\title{
Entre el Ciborg y el Barroco: un Entrecrucede Caminos Tecno-Científicos ${ }^{1}$
}

\author{
Juan R. Coca ${ }^{2}$ \\ Jesús A. Valero Matas ${ }^{3}$ \\ Alejandra Solano ${ }^{4}$
}

\begin{abstract}
RESUMEN: El presente texto pretende analizar la sociedad actual. En ella la tecnociencia tiene una presencia importante y ésta, a su vez, no es muy diferente con respecto a las demandas intelectuales, culturales y sociales del Barroco. Es decir, en la sociedad actual podríamos decir que sucede lo mismo, interesa más obtener resultados, que se nos asombre, más que comprender el cómo funciona o se realizan. Si bien, como aconteció en el Barroco, sectores de la sociedad emprendieron acciones para dotar de más identidad y cultura a la sociedad e ir en contra de lo institucionalmente establecido. La sociedad actual, se puede decir que es una prolongación del barroco, y nos encontramos en el neobarroco. Este al igual que en el pasado se rebela contra el sistema que sólo está preocupado por la acumulación de dinero, así como contra la ausencia de libertad y, por tanto, contra la represión y la violencia institucionalizada. En definitiva, con este artículo se trata de dar una visión desde la hermenéutica de las similitudes del Barroco con nuestra sociedad actual.
\end{abstract}

PALABRAS CLAVE: Barroco. Sociología. Ciborg. Ciencia. Hermenéutica.

\section{INTRODUCCIÓN}

El barroco es una etapa de la historia iberoamericana de gran relevancia. De hecho, el Siglo de Oro español se enmarca, precisamente, en este momento histórico. El presente trabajo parte de la premisa de que en el mundo iberoamericano existe una tradición de reflexión y pensamiento diferencial e identitária particular. A su vez se pretende ahondar e incrementar el conocimiento existente sobre el barroco iberoamericano y como éste podría

\footnotetext{
${ }^{1}$ Este trabajo es parte del Proyecto de Innovación Docente 13-15 "Aplicación de las TEP en el fomento del espíritu crítico y democrático del alumnado universitario" financiado por la UVa. Dirigido por el prof. Juan Romay Coca, y del Proyecto financiado por la UVa (PIP) Estudio de los factores determinantes en la elección de los estudios de ciencias, humanidades y ciencias sociales en los Universitarios españoles, dirigido por el prof. Jesús A. Valero Matas.

${ }^{1}$ http://dx.doi.org/10.1590/S0101-317320160003000010

${ }^{2}$ Facultad de Educación. Campus "Duques de Soria”. Soria (España). Tfno.: 0034975129234

E-mail: juancoca@soc.uva.es

${ }^{3}$ Dpto. Sociología y Trabajo Social. Universidad de Valladolid.

${ }^{4}$ Dpto. Sociología y Trabajo Social. Universidad de Valladolid.
} 
proporcionarnos una nueva perspectiva sociológica. Para ello conjugamos diversos trabajos que, a nuestro juicio, tienen gran relevancia sociológica actual: el actual proceso de ciborgización y la necesidad del fomento de la comunidad en nuestra sociedad.

En este sentido diversos autores han indagado, de un modo extremadamente interesante y sugerente, en la apuesta del barroco como elemento específicamente nuestro. De hecho, recientemente, Carlos Soldevilla Pérez en su Ser barroco ha propuesto

[...] rescatar la dimensión aurática del barroco, sacándola del desván histórico para instalarla en otra historia, la historia de la actualidad. Pues la cultura barroca posee excelentes valores, como son, entre otros: el autodominio prudente; su apuesta por el tiempo pausado y rememorativo; el énfasis en la estética y en la espiritualidad; el desasimiento de la materialidad de las cosas; su atención y cuidado hacia el arte de la mirada; el autoconocimiento a través de las cartografías del sueńo y el arte; su comunitarismo de proyectos y referencias compartidas; y, por último, la reivindicación -no miserabilistade la cultura popular; todos ellos valores alternativos a los de una modernidad global y materialista. (SOLDEVILLA, 2013, p. 35).

Para la consecución de nuestros objetivos es necesario repensar el concepto de sociedad. No obstante, si partimos del hecho de que el barroquismo es algo característico del mundo iberoamericano y concretamente del contexto español, por ende, consideramos que es conveniente recurrir al pensamiento español para profundizar en el término sociedad. Para ello recurriremos, en esta ocasión, al filósofo espańol Xavier Zubiri y lo vincularemos con el barroquismo. Aún así, somos muy conscientes que atribuirle a Zubiri ciertas características de barroquismo es arriesgado. No obstante, consideramos que -como hipótesis inicial- este autor asume algunos elementos propios del mismo y que, efectivamente, parece que podría estar situado en lo que podría denominarse como pensamiento social iberoamericano. A lo largo del presente trabajo contrastaremos esta hipótesis. Pero, además, ahondaremos y reinterpretaremos el futuro que se nos abre a través de esta tradición barroca y veremos si puede ser relacionada con la nueva concepción sobre el ciborg iniciada por Donna Haraway. En este sentido también tomaremos como nuestra la idea de que la artificiosidad del barroco ha traído consigo la configuración cibórgica del ser humano y de su sociedad. No hay que dejar de lado que estas dos épocas destacan por la labor de investigación en automatismos y "nuevas tecnologías". Autores como Leonardo da Vinci, Juliano Turriano o 
René Descartes se convirtieron en los gurús de una época convulsa atraída por múltiples avances, entre los que se podría destacar el campo de la relojería, lo que llevo a esta época barroca a ser conocida como la edad de oro en la que los mejores y más perfectos autómatas se realizarían. Su desarrollo, dominado por el carácter científico, ponía de relieve la obsesión por intentar reproducir lo más fielmente posible los movimientos y comportamientos de los seres vivos.

Por otro lado, también se produje una gran pasión por los ingenios mecánicos en el Barroco que llegaría a tal extremo que no son pocas las menciones, aunque son escasos los testimonios gráficos, a la utilización de artificios mecánicos es fiestas locales y religiosas de las época, como ejemplo las fiestas del Corpus Christi de 1677 en Córdoba relatadas e ilustradas por el maestro Ginés de Godoy.

En la actualidad no tendríamos nada más que hacer que cambiar relojería por tecnología informática y el resultado sería equivalente, robots insectos, simulación y creación digital de entornos y actores digitales. Al igual que en el Barroco los autómatas y diversos mecanismos hacían creer que lo desconocido había sido descubierto, solamente se mostraban los resultados o las acciones, actualmente podríamos decir que sucede lo mismo, interesa más obtener resultados, que se nos asombre, más que comprender el cómo funciona o se realizan.

Este artículo se ha configurado a partir de un análisis documental sobre el barroquismo y su relación con la sociedad. Nuestra intención no ha consistido en hacer un análisis extensivo y descriptivo de todos los textos. Sino que nos hemos aproximado a los trabajos que, a nuestro juicio, aportar un mejor conocimiento sobre los objetivos establecidos en este trabajo de investigación y que nos permita el pertinente contraste de hipótesis. Nuestra investigación documental tiene carácter histórico y cultural que, como bien afirma Mills (1999) en La imaginación sociológica nos permite amplificar nuestra visión gracias al incremento de su campo de observación abarcando un período histórico suficientemente amplio como para captar el significado que trata de comprender y explicar.

\section{BARROCO, NEOBARROCO Y CIBORG}

El Barroco desarrolla dos tipos de estrategias de conocimiento del ego: 
[...] unas más propias de la sociedad cortesana, es decir, del contexto social y político en que se desenvuelve la vida de los individuos y otras más típicamente religiosas, de construcción de un "yo interior", volcado en el autoconocimiento y dominio de sí con fines transcendentes. (GONZÁLEZ, 2005, p. 141).

Este doble camino será recurrente en este trabajo y, además, tendrá gran utilidad para la consecución de nuestros objetivos. El barroco se mantiene presente hoy en día en lo que se ha dado por llamar neobarroco, que ha sido estudiado especialmente a nivel lingüístico y que puede ser concebido “[...] como un resurgimiento crítico y estilizado de algunos elementos del barroco histórico como la exuberancia, la ornamentación y el conceptismo en la literatura y las artes plásticas del último medio siglo." (KULAWIK, 2012). Pero además, el Barroco puede llegar a concebirse -tal y como veremos más adelante- como un modo de rebelarse contra el sistema que sólo está preocupado por la acumulación de dinero, así como contra la ausencia de libertad y, por tanto, contra la represión y la violencia institucionalizada. Durante el barroco y en la actualidad hay que tener presente que la propia ciudad se convertía en una compleja escenografía que disfrazaba una realidad para imponerse como instrumento didáctico y de poder.

Barroco o neobarroco son términos que muestran, siguiendo a D’Ors (1964) o Carpentier. El primero consideraba que había manifestaciones artísticas semejantes al barroco en todos los periodos de la historia. Carpentier, en cambio, considera que el barroco mantiene su horror vacui, su rechazo a la nuda superficie y también a la linealidad y geometricidad excesiva. En el barroco, para Alejo Carpentier (1976), se van desarrollando los elementos decorativos en torno a unos "núcleos proliferantes". Esta idea literaria y, en general, artística tiene cierto correlato social en los imaginarios sociales. Estos núcleos proliferantes pueden ser reconceptualizados como elementos imaginarios que funcionan socialmente como pivotes de irrealidad a través del cual la población va estructurando los elementos "ornamentales" de la sociedad. De ahí que, a nuestro juicio, asumimos esta perspectiva y consideramos que - desde una perspectiva sociológica- no existe una diferenciación clara entre barroco y neobarroco (salvo la obvia distinción histórica).

En este sentido es conveniente advertir que el concepto de barroquismo actual o de neobarroco ha tenido repercusión en literatura, filosofía y en el pensamiento en general. Ello implica que existan ciertos problemas de 
identificación. De ahí que Calabrese, apuesta por centrarse en una serie de "síntomas" que serán los que nos permitan delimitar neobarroco. A saber, límite, exceso, detalle, fragmento, ritmo, repetición, inestabilidad, metamorfosis, nudo, laberinto y desorden. Recuérdese que, como acabamos de decir, para nosotros no existe una distinción clara entre barroco y neobarroco, al considerarlo como un elan o un eón epocal.

Esta idea nos indica que vivimos, desde una perspectiva sociológica, en el mundo líquido del que nos ha hablado Zygmunt Bauman en diversas ocasiones. En él existe una tensión extrema del límite (CALABRESE, 1989) y una tendencia al riesgo o al abismo, propia de dicha tensión del límite. Viendo esto podemos entender con facilidad la excentricidad, el escándalo, la provocación, la diseminación, la fragmentación, lo caótico, etcétera que huyen del mundo sólido, cerrado y lógico.

En esta convulsión por transgredir límites, por fragmentar y alterar se ha configurado lo que se ha dado en llamar como ciborg. Este concepto ha sido altamente conocido en la academia gracias a los trabajos de Donna Haraway (1991) quien lo definió como un híbrido entre un ser humano y una máquina creada ex novo y relativamente coincidente con la entidad imaginaria propia de la ciencia ficción. Otro enfoque es aquel que entiende que el concepto de ciborg desde una perspectiva más amplia hace referencia al superhombre de Nietzche. Una visión todavía más amplia la han expresado autores como Andrés Moya, Francesc Mestres, Josep Vives o Igor Sádaba quienes muestran una concepción del ser humano relacionada con las transformaciones tecnológicas, del tipo que sean, y que puede hacer referencia también a los nuevos procesos comunicacionales, a la incorporación de nanotecnologías en el cerebro, etc. Como bien exponen Mestres y Vives, el concepto de ciborg está vinculado a la idea de aumentación de las capacidades humanas. De ahí que la ingesta de medicamentos, la cirugía plástica, la implantación de "órganos bioartificiales", el uso de células madre, las transfusiones, etc. son buenos ejemplos de dicha aumentación.

Como acabamos de ver la ciborgización es, sin ninguna duda, una superación de los límites humanos basada en la recreación de un nuevo ser humano. La idea fundamental de esto es la materialización de lo que podríamos denominar como humano ficcional. Es decir, a nuestro juicio, los humanos buscamos materializar una serie de imaginarios sociales. Para ello, en muchas ocasiones, debemos transgredir nuestra naturaleza en base 
a procesos de hipertrofia de alguna de nuestras características o, incluso, de nuestro entorno (naturaleza, ciudades, etc.).

Tal vez por relatarlo con la época barroca podríamos asemejarlo con los "jugadores de ajedrez" o también llamado "El Turco" creado por Wolfgang von Kempelen, que aunque de finales del siglo XVIII vendrían a ser la culminación de la alianza hombre-máquina de este período. Una de las mayores atracciones de la época, heredada por Johan Mazel, de la que decían era "invencible", pero que, sin poder afirmarlo completamente, era la unión de una mecanismo ingenioso controlado o manejado desde su interior por una persona y así parece demostrarlo el que tras la muerte de su asistente en Cuba no volviera a ser puesta en funcionamiento.

En épocas posteriores nos referiríamos a Leonardo Torres Quevedo como su sucesor, aunque la culminación de estos intentos de crear una inteligencia artificial inspirada en el ser humano y que pueda superarla la tenemos en el superordenador Deep Blue. Ahora bien, este proceso de ciborgización humana también ha tenido su contrapartida. De hecho Cornago (2004) nos indica que los avances en la biología molecular, la neurobiología, etc. han traído consigo una manera distinta de entender tanto la materia como la vida y, además, generó el nacimiento de corrientes críticas diferentes.

Según lo expresado, el neobarroco (o si se prefiere el barroco actual) parece no recuperar el humanismo y la trascendentalidad existente en el barroco (el convencionalmente admitido) e incluso podríamos decir, siguiendo a Calabrese, que el neobarroco sólo conserva una línea deshumanizadora, maquinal y ficcional de lo humano y de la humanidad. Dicho de otro modo, en nuestro mundo actual se han mantenido ciertos elementos propios del Barroco. El problema es que se han hipertrofiado ciertos elementos deshumanizadores y artefactualizadores del barroco sin el contrapeso humanístico que existe en el Barroco convencional. De hecho, como nos muestra Mauricio Beuchot (2005, p. 143) "[...] también hay cosas del barroco originario que creo que podrían servirnos para no tener el camino tan perdido”. Este es, precisamente, lo que vamos a tratar a partir de ahora.

\section{Barroco y ANALOgía}

Antes de continuar vamos a pararnos por un momento en el barroco que ha desarrollado en una zona de España: Galicia. En esta región, situada 
en el noroeste de la Península Ibérica, esta corriente ha tenido una relevancia enorme. Torres Queiruga (1984), uno de los grandes filósofos y teólogos gallegos, afirma que desde comienzos del siglo XVII se produce un resurgir cultural en esta región de España. Este proceso, identificado como Barroco, se produce en contraste con una gran pobreza socio-económica. El Barroco en Galicia y también en Portugal ha tenido una importancia cultural. Posiblemente en ambas regiones no ha habido otra corriente cultural de tanta importancia como esta.

El Barroco gallego, según uno de sus máximos exponentes: Otero Pedrayo (1982), es más que un movimiento es una hibridación de la cultura y de la naturaleza. Este autor consideró que el Barroco es un concepto repleto de matices que hace mención a una realidad detallista, minuciosa, naturalizada y, evidentemente, religiosa.

El barroco presentaba un interesante enfoque sobre la problemática del ego que, a nuestro juicio, se mantiene en la actualidad en la sociedad postmoderna (y neobarroca). Como nos informa José M. González García (2005) la sociedad barroca era muy conflictiva y estaba muy dividida; de ahí que la identidad barroca (el self o ego barroco) se configura en este marco de crisis. Ahora bien, pese a dicho establecimiento identitario en base a fenómenos críticos, la construcción de dicha identidad en la actualidad no está vinculada a las crisis del ego basadas en los procesos de reconocimiento de los que nos habla Goffman (2009) sino que se configura en el marco de otra crisis; una lucha por la adquisición de conocimiento. Dicho de otro modo, para Goffman (2009) las personas ubicamos a los demás, de manera cognitiva, a través de procesos de reconocimiento. De esta manera nos otorgamos unos a otros una determinada identidad social (comunitaria) o personal particular (íntima). En este sentido Erving Goffman afirma que existe un reconocimiento cognitivo que hace mención de un acto de percepción individual y un reconocimiento social.

En cambio en el Barroco el ego se mueve en un entorno más plural y complejo. El auto-conocimiento, por tanto, será el de la naturaleza, el de los demás y el de uno mismo. En esta búsqueda y lucha por el auto-conocimiento sucedía un control absoluto de las emociones internas, puesto que el ego debía ser una fortaleza inexpugnable que tras una fase de auto-conocimiento podía, entonces, relacionarse con los demás.

En consecuencia el sujeto que se constituye en el autodominio prudente, sabe conocerse y templarse, para ulteriormente tender a relacionarse con aquellos individuos y grupos con los que comparte objetivos, planes y fines 
de vida, esto es, con aquellos que conforman, desde sus afinidades electivas, una comunidad de estilo de vida. (SOLDEVILLA, 2013, p. 105).

En definitiva en un mundo en crisis, como el Barroco, sólo el autodominio prudente (phrónesis) nos permitirá relacionarnos con los demás de una manera adecuada y socialmente aceptable. Esta necesidad de la prudencia en los procesos de relación social puede ser recobrada y convertirse en alternativa en la sociedad actual. Esto es lo que ha planteado recientemente Soldevilla (2013) y durante los últimos años el profesor Mauricio Beuchot en sus trabajos sobre la analogía y la prudencia. Ahora bien, en el barroco iberoamericano dicha prudencia devino en máscara pública. En cambio, puede entenderse la prudencia barroca como una relación social sosegada en la que todos buscan no incordiar a los demás. Por esta razón decíamos antes el cierto Barroco es una alternativa posible que puede ser defendida en la actualidad como un sendero por el que nos podemos desviar en nuestro mundo tan condicionado tecnológicamente y tan hipertrofiado en los procesos comunicacionales (mass media, redes sociales, etc.).

Beuchot (2005) nos demuestra que el barroco fue un momento histórico sumamente analógico a través del estudio de la obra de Foucault, Gracián y los místicos de la época. Beuchot a lo largo de sus diversas obras nos indica que el concepto helénico y medieval de analogía tiene gran vigencia en la actualidad a nivel sociológico, histórico, epistemológico, ético, etc. Gracias a él podemos articular una concepción humana más humilde y, en línea con el barroquismo de Gracián, más autoconsciente y prudente.

Foucault (1968: 54) en Las palabras y las cosas hace mención de Don Quijote como uno de los grandes personajes análogos; mostrándonos, entonces, como la analogía está claramente presente en Cervantes. De hecho, afirma Foucault(1968, p. 54) acerca de este personaje:

Todo su camino es una búsqueda de similitudes: las más mínimas analogías son solicitadas como signos adormecidos que deben ser despertados para que empiecen a hablar de nuevo. Los rebańos, los sirvientes, las posadas se convierten de nuevo en el lenguaje de los libros en la medida imperceptible en que se asemejan a los castillos, a las damas, a los ejércitos. Semejanza siempre frustrada que transforma la prueba buscada en burla y deja indefinidamente vacía la palabra de los libros. 
Asimismo, este filósofo nos muestra que Don Quijote es una mezcla de locura y poesía. De locura como homosemantismo, dice, pero también de poesía como alegoría. Es decir, dice Foucault (1968, p. 56), el loco “[...] junta todos los signos y los llena de [...] semejanza", mientras que "el poeta hace llegar la similitud hasta los signos de hablan de ella". Es decir, estamos, como hemos visto en el apartado anterior, en un mundo ficcional en el que la solidez y la liquidez están en constante lucha. Dicho de otro modo, Don Quijote se sitúa constantemente en los límites y se convierte él en uno de los personajes más análogos y paradójicos de la literatura española.

Creemos que es evidente que esta contienda se mantiene en esta actualidad neobarroca o postmoderna. El debate y la lucha constante entre el mundo de la locura y el de la poesía están presentes en nuestra sociedad. De ahí que la aproximación a la realidad social no pueda ceñirse a la mera adquisición de datos e información cuantitativa. Sino que también se hace necesaria una aproximación hermenéutica, o si se prefiere por algunos literaria, a nuestro mundo social. Este tema, de gran relevancia sociológica, no puede ser tratado en profundidad en este trabajo. No obstante es importante dejar constancia que el planteamiento barroco también nos abre la puerta a una epistemología más comprensiva.

El Barroco, dada su excentricidad, mostró una relativa ausencia de centro de poder. Por ello, el Barroco Iberoamericano implica un fuerte mestizaje enraizado en las prácticas sociales (SANTOS, 2012). Si esta idea la conducimos al mundo de la investigación y del saber nos encontramos, en parte, con una resistencia a las pretensiones de dogmatismo de una determinada razón instrumental (SOLDEVILLA, 2013, p. 146) e incluso del racional-positivismo al uso. Asimismo, tal y como nos muestra Mauricio Beuchot (2005) el Barroco al que estamos haciendo mención, es decir el nuestro, también está vinculado con la espiritualidad y el misticismo. Por esta razón, nos dice, el Barroco fue también una época de grandes místicos.

Esta doble idea de contradogmatismo racional-positivista y de espiritualidad parece haberse mantenido, como corriente subyacente, a lo largo de la historia de la investigación filosófica y sociológica iberoamericana. De hecho, podemos poner como ejemplo el trabajo de estudiosos tales como María Zambrano, quien defendía la sabiduría frente al método, Urbano González Serrano, quien defendía la complejidad social y por tanto no una investigación limitada y limitante sino amplia, Xavier Zubiri, con su inteligencia sentiente, Adolfo Posada o Vicente Santamaría de Paredes, quienes consideraban que la 
sociología tiene relación con la metafísica, Unamuno y Ferrater Mora, como unos de los pensadores más paradójicos y próximos al límite, etc.

Esto nos indica que podemos apostar por un neobarroco no postmoderno donde la humanización de la sociedad y de la persona sean los objetivos fundamentales de nuestro trabajo. Ello lo logramos bien porque es posible un conocimiento altermoderno no regido por una razón instrumental dogmática y ensimismada, bien porque existe una trascendencia (sea cual sea) que nos muestra un horizonte al que podemos aproximarnos.

\section{El CONCEPTO DE SOCIEDAD EN ZUBIRI}

Xavier Zubiri ha desarrollado en su filosofía un enfoque que, a nuestro juicio, tiene un gran interés sociológico y consideramos que podría tener cierta relación con el pensamiento barroco. Analicemos esto y comprobemos si es así.

Para el pensador vasco el ser humano está vertido a sí mismo y a los demás. Dicha versión, nos dice, es de convivencia pero no en abstracto, sino a personas concretas. Este enfoque nos recuerda al planteamiento unamuniano el cual también mostraba un alto nivel de concreción. Para Zubiri, la sociedad no tiene vida. "Lo único que hay son las vidas de cada cual socialmente tomadas." (ZUBIRI, 1998, p. 302) El concepto de versión, al que estamos haciendo referencia, ha sido prácticamente olvidado en sociología haciendo que esta ciencia se haya restringido a la actividad conjunta de un determinado grupo social y no a los elementos relacionales de la misma. Por suerte la sociología relacional intenta modificar esto. Esta versión humana hace que la propia persona sea una co-realidad constitutiva.

Los hombres son constitutivamente co-reales y constitutivamente no quiere decir que lo sean porque no tienen más remedio, sino que lo que es cada uno es co-real con otros por razón de su propia realidad. La convivencia es un carácter específico de la realidad propia de la sustantividad humana en cuanto tal. No es algo que derive de ello. Y, por consiguiente, el verdadero problema de la convivencia estriba en que se nos diga en qué medida y en qué forma los otros forman parte de mi propia realidad y, recíprocamente, en qué medida yo formo parte de la realidad de los otros. (ZUBIRI, 2006, p. 51).

Zubiri (1998) pretende decirnos que todos y cada uno de nosotros, al vivir en sociedad, tiene un hábito de alteridad que tiene una estructura formal, la convivencia, una estructura dinámica, la expresión, y una estructura fundante 
que le da carácter fundante. Dicho de otro modo, el ser humano está vertido a los demás y gracias a ello, convivirá con otras personas. Esta convivencia no será estática sino que se expresará de maneras muy distintas. Por último, el hecho de que estemos vertido a los demás hace que nos convirtamos en posibilidad para los demás. Ahora bien:

Se trata de mi propia vida, en tanto que modificada por la vida de los demás, que para ellos es también propia. Pues bien, la manera positiva como la vida de los demás afecta a mi propia vida en tanto que propia, es justamente la apropiación. Solamente en la medida en que el hombre esté apropiándose la vida de los demás, cada uno es cada cual. (ZUBIRI, 1998, p. 305).

A lo que añade: "Por mucho que yo me sienta identificado con la vida de los demás, jamás me apropiaré de la vida del otro, sob pena de que el otro desapareciera." (ZUBIRI, 1998, p. 305) Vemos que, tras lo dicho, Zubiri se aleja de la concepción sociológica actual acerca de la representación de las personas en sociedad. Al contrario, configuramos nuestra propia vida a través del conocimiento - la apropiación - que vamos adquiriendo sobre los otros. Este conocimiento del otro nos abre un mundo de posibilidades. En este sentido, Zubiri rechaza la concepción negativa de la sociedad y de los otros. El otro se convierte entonces en posibilidad inexorable.

No obstante, esta indefectibilidad posibilitante tiene una dimensión negativa proveniente de la finitud de posibilidades que me ofrece el otro. De tal modo que quedan fuera otras posibilidades que nos podría ofrecer otra persona. Además de lo dicho es conveniente advertir, siguiendo en cierto modo lo dicho en el apartado anterior, que el otro es un analogon en referencia a Husserl. Pero el que el otro sea un analogon puede significar que la otra persona sea un otro-como-yo o bien un otro-que-yo. En el primer sentido estaremos en el marco de la analogía, mientras que en el segundo estaremos en lo opuesto (ZUBIRI, 1998, p. 232).

Por lo tanto, el otro será posibilidad fáctica y no meramente formal cuando sea otro-como-yo convirtiéndose en un análogo. Pero para que esto suceda es necesario que los demás se introduzcan en el ego vinculándose y otorgándoles unos modos humanos de actuación. 


\section{ConCLUSIÓN}

En el presente trabajo, desde una perspectiva teórica, hemos analizado como la sociedad actual puede ser interpretada como una prolongación imaginaria (es decir mantiene una serie de imaginarios sociales previos) del Barroco. Es cierto que puede concebirse la ilustración como ruptura del Barroco, ahora bien (siguiendo a los autores citados anteriormente) podemos considerar que en la historia humana se han mantenido, de un modo $\mathrm{u}$ otro, ciertos elementos imaginarios o significativos fundamentalmente en el contexto Iberoamericano.

El Barroco, o neobarroco, presenta un elemento de rebelión y transgresión sistémica debido, en buena medida, a su carácter naturalista y espiritual. De ahí que los imaginarios sociales barrocos se contraponen a este sistema actual que parece estar solamente preocupado por la acumulación de dinero, incremento del beneficio empresarial, etc. Así mismo, el sistema parece también mostrar una deriva encaminada no tanto al incremento de las posibilidades humanas, de la libertad y de la relacionalidad entre personas en el sentido zubiriano, sino más bien en dirigida al polo opuesto. De ahí que surjan elementos teóricos y sociales que buscan reducir esto, contrarrestas la situación social y mejorar la vida de las personas. Todo ello, si lo pensamos bien, nos recuerda al barroco, parece que nos hemos introducido en un mundo del que nos está costando salir. Ello nos permite confirmar las hipótesis de nuestro trabajo. En definitiva, el imaginario social Barroco es recurrente y esto nos permite reinterpretar la sociedad desde una nueva perspectiva que todavía no se ha hecho. Convendría, entonces, hacer una nueva hermenéutica sociológica de la sociedad actual bajo esta perspectiva.

COCA, Juan R.; MATA, Jesús A. Valero; SOLANO, Alejandra. Among the cyborg and the baroque: a road techno-scientific crisscross. Trans/form/ação, Marília, v. 39, n. 3, p.177-190, Jul./Set., 2016.

\footnotetext{
ABSTRACT: This paper aims to analyze current society, where techno-science has a major presence while at the same time not differing greatly from that of the Baroque era with respect to intellectual, cultural, and social demands. As in the Baroque era, we are today more interested in getting results than in understanding how things works or how things are done. Also as in the Baroque era, sectors of society are taking action to encourage more identity and culture in society and are going against what is institutionally established. Today we can say that our society is an extension of the Baroque, and that we are in the neo-Baroque era. As in the past, there is now a rebellion against a system that is
} 
only concerned with the accumulation of money, as well as a rebellion against the lack of freedom and therefore against repression and institutionalized violence. In short, this article aims to present, from the point of view of hermeneutics, similarities between our current society and that of the Baroque era.

KEYWORDS: Baroque, sociology, cyborg, science, hermeneutics

\section{REFERENCIAS}

BEUCHOT, M. En el camino de la hermenéutica analógica. Salamanca: San Esteban, 2005. CALABRESE, O. La era neobarroca. Madrid: Cátedra, 1989.

CARPENTIER, A. Razón de ser. Caracas: Universidad Central de Venezuela, 1976.

CORNAGO, O. Nuevos enfoques sobre el Barroco y la (Pos)Modernidad (a propósito de dos estudios de Fernando R. de la Flor). Dicenda: Cuadernos de Filología Hispánica, n. 22, p. 27-51, 2004.

D’ORS, E. Lo barroco. Madrid: Aguilar, 1964.

FOUCAULT, M. Las palabras y las cosas. México: Siglo XXI, 1968.

GOFFMAN, E. La presentación de la persona en la vida cotidiana. Madrid: Amorrurtu, 2009.

GONZÁlEZ G. J. M. Metáforas e ironías de la identidad barroca. In: ARIÑO VILLARROYA, A. (Ed.). Las encrucijadas de la diversidad cultural. Madrid: Centro de Investigaciones Sociológicas, 2005. p. 139-158.

GONZÁLEZ SERRANO, U. La sociología cientifica. Madrid: CIS-BOE, 2003.

HARAWAY, D. J. A Cyborg manifesto: science, technology, and socialist-feminism in the late twentieth century. In: HARAWAY, D. J. Simians, cyborgs and women: the reinvention of nature. New York: Routledge, 1991. p. 149-181.

KULAWIK, K. Travestir (en) los límites: identidades transitivas en el discurso neobarroco de la neovanguardia latinoamericana. Amerika [En línea], n. 7, 2012.. Accesible en: <http:// amerika.revues.org/3366 >. Consultado el 02 sept. 2013. (DOI: 10.4000/amerika.3366).

MENDEZ RODRIGUEZ, L. Sobre autómatas en las fiestas del Corpus Christi en 1677. Laboratorio de Arte, n. 18, p. 209-220, 2005.

MILLS, C. W. La imaginación sociológica. México: FCE, 1999.

MESTRES, FRANCESC Y VIVES-REGO, J. Precisiones interdisciplinares y conceptuales de los términos cyborg, clon humano y robot. Ludus Vitalis, v. XIX, n. 35, p. 235-238, 2011.

OTERO PEDRAYO, R. Ensaio histórico sobre a cultura galega. Vigo: Galaxia, 1982.

POSADA, A. Principios de sociología. Madrid: Daniel Jorro, 1929. V. 2

SANTAMARÍA DE PAREDES, V. El concepto de organismo social. Madrid: Fernando Fe, 1896. SANTOS, B. de S. Una epistemología del sur. Buenos Aires: CLACSO; Siglo XXI, 2012. 
SOLDEVILLA PÉREZ, C. Ser barroco: una hermenéutica de la cultura. Madrid: Biblioteca Nueva, 2013.

TORRES QUEIRUGA, A. La religión y la Iglesia, In: VV.AA. Los gallegos. Madrid: Istmo, 1984. p. 479-501.

UNAMUNO, M. Del sentimiento trágico de la vida en los hombres y en los pueblos. Madrid: Biblioteca Nueva, 1999.

ZUBIRI, X. Sobre el hombre. Madrid: Alianza-Fundación Xavier Zubiri, 1998.

. Tres dimensiones del ser humano: individual, social, histórica. Madrid: AlianzaFundación Xavier Zubiri, 2006.

Inteligencia sentiente. Madrid: Madrid, 2004.

Recebido em 06/11/2015

Aceito em 20/05/2016 